\title{
Imaging features of pulmonary involvement in a case of systemic amyloidosis: A classic case
}

Pattharapong Saneha, M.D.

Thanarak Thongsuk, M.D.

Laksika Bhuthathorn, M.D.

From Department of Radiology, Faculty of Medicine, Prince of Songkhla University, Songkhla, Thailand

Address correspondence to P.S. (e-mail: peetpat34@gmail.com)

Received 8 December 2020; revised 15 December 2020; accepted 16 December 2020 doi:10.46475/aseanjr.v21i3.99

\section{Abstract}

Amyloidosis is a disease caused by pathologic extracellular deposition of abnormal insoluble proteins throughout the body[1]. Pulmonary amyloidosis is a form of amyloid deposition confined in the lung parenchyma and may cause airway obstruction, dysphagia, and chronic pleural effusions, often with nonspecific chest imaging findings[1,2].

A 56-year-old male with underlying light chain multiple myeloma and systemic amyloidosis presented with fever for two days without dyspnoea or cough. Further chest imaging revealed nonspecific findings including consolidations, ground-glass opacities, interlobular septal thickening in both upper lobes, and bilateral pleural effusions; a diagnosis of pneumonia with pulmonary oedema was made. After the patient failed to respond to treatment, bronchoscopy with tissue biopsy was performed for unresolving pneumonia. Histopathological results were consistent with pulmonary amyloidosis.

Keywords: Pulmonary amyloidosis, Systemic amyloidosis, Multiple myeloma. 


\section{Introduction}

Amyloidosis is a rare disease caused by pathologic extracellular deposition of abnormal insoluble proteins throughout the body[1]. Pathogenesis of amyloidosis is characterised by protein misfolding disorders, resulting in accumulation and aggregation of this nonfunctional and toxic protein which causes damage to cells and tissues[3]. The disease may be classified as a primary or secondary disease, with primary amyloidosis being more common. Secondary amyloidosis may be caused by a variety of pathologic processes such as chronic infection, inflammation, or underlying malignancies[1].

Amyloidosis involves various organs including lungs, heart, kidneys, liver, soft tissues, the peripheral and/or autonomic nervous system, and the gastrointestinal tract[4]. The clinical symptoms of pulmonary amyloidosis may range from asymptomatic to nonspecific symptoms such as progressive dyspnoea, cough, wheezing, and respiratory failure[5].

Pulmonary involvement of amyloidosis may manifest as nodular parenchymal and diffuse alveolar septal forms, both of which have nonspecific radiological findings. Imaging findings of nodular parenchymal amyloidosis includes solitary or multiple pulmonary nodules which may, albeit rarely, calcify and cavitate[1]. These findings mimic a variety of other diseases, including granulomatous infections and malignancies, making a diagnosis based on imaging findings alone challenging and biopsy is often required for a definitive diagnosis.

Diffuse alveolar septal amyloidosis is less commonly found, but more clinically important as patients usually have a far worse prognosis compared to a nodular parenchymal subtype and are more likely to develop pulmonary hypertension and respiratory failure. Imaging findings of diffuse alveolar septal amyloidosis includes reticulonodular opacities, interlobar septal thickening, and confluent consolidations with basal and peripheral predominance[1]. 
Pleural involvement of amyloidosis most commonly manifests as pleural effusions, and airway involvement may manifest as long-segment submucosal plaques in the airways [1]. Pulmonary amyloidosis may cause airway obstruction, dysphagia, and chronic pleural effusions[2].

In this article, we present a classic case of pulmonary amyloidosis manifesting as nonspecific radiological findings of the chest in a case initially diagnosed with pneumonia, but subsequently diagnosed pulmonary amyloidosis through bronchoscopy with tissue biopsy performed for unresolving pneumonia.

\section{Case Summary}

A 56-year-old man with underlying International Staging System (ISS) stage II light chain multiple myeloma, biopsy-proven systemic amyloidosis, and MRI-proven cardiac amyloidosis, presented with fever for two days without dyspnoea or cough. Physical examination reveals that the patient had a fever, hypotension, and tachypnoea. An examination of the lungs revealed bilateral diffuse crepitation. Laboratory results showed mild anaemia (Hemoglobin 9.1 g/dl, Hematocrit 27.8\%), with normal white blood cell and platelet counts. His chest radiograph reveals diffuse patchy opacification with air bronchogram in both lungs, predominantly in both upper lobes (figure 1).

The initial problem list included pneumonia with septic shock in an immunocompromised host, suspected pulmonary oedema, and his underlying medical conditions. A provisional diagnosis of bacterial pneumonia was made, while differential diagnoses included opportunistic infections such as Pneumocystis jiroveci pneumonia (PJP), pulmonary tuberculosis, and other fungal infections. He was admitted for antibiotic treatment and further laboratory investigations. 
Sputum cultures found Pseudomonas aeruginosa, and the PCR test was positive for Pneumocystis jiroveci; a diagnosis of bacterial pneumonia and PJP was made. After initial treatment with antibiotics and diuretics, the patient's clinical symptoms failed to improve and further diagnostic bronchoscopy for evaluation of unresolving pneumonia was indicated.

A venous phase chest computed tomography (CT) was performed for preprocedural evaluation prior to bronchoscopy by the pulmonologist. The patient's chest CT showed consolidations, ground-glass opacities, and interlobular septal thickening, predominantly in both upper lobes (figure 2). Bilateral pleural effusions were also detected. These nonspecific imaging findings support both diagnoses of pneumonia and pulmonary oedema and the patient underwent diagnostic bronchoscopy as planned.

Bronchoscopy revealed normal bronchi without endotracheal mass, and bronchoalveolar lavage with transbronchial tissue biopsy of the posterior segment of the right upper lobe was done. Histological examination revealed interstitial thickening with deposition of pink amorphous material. Congo red stain revealed apple-green birefringence in polarised light (figure 3); findings were consistent with amyloid deposits. The patient was therefore diagnosed with pulmonary amyloidosis.

After further treatment with antibiotics and diuretics, the patient's clinical condition slowly improved, and he was subsequently discharged with antibiotics for PJP prophylaxis. 


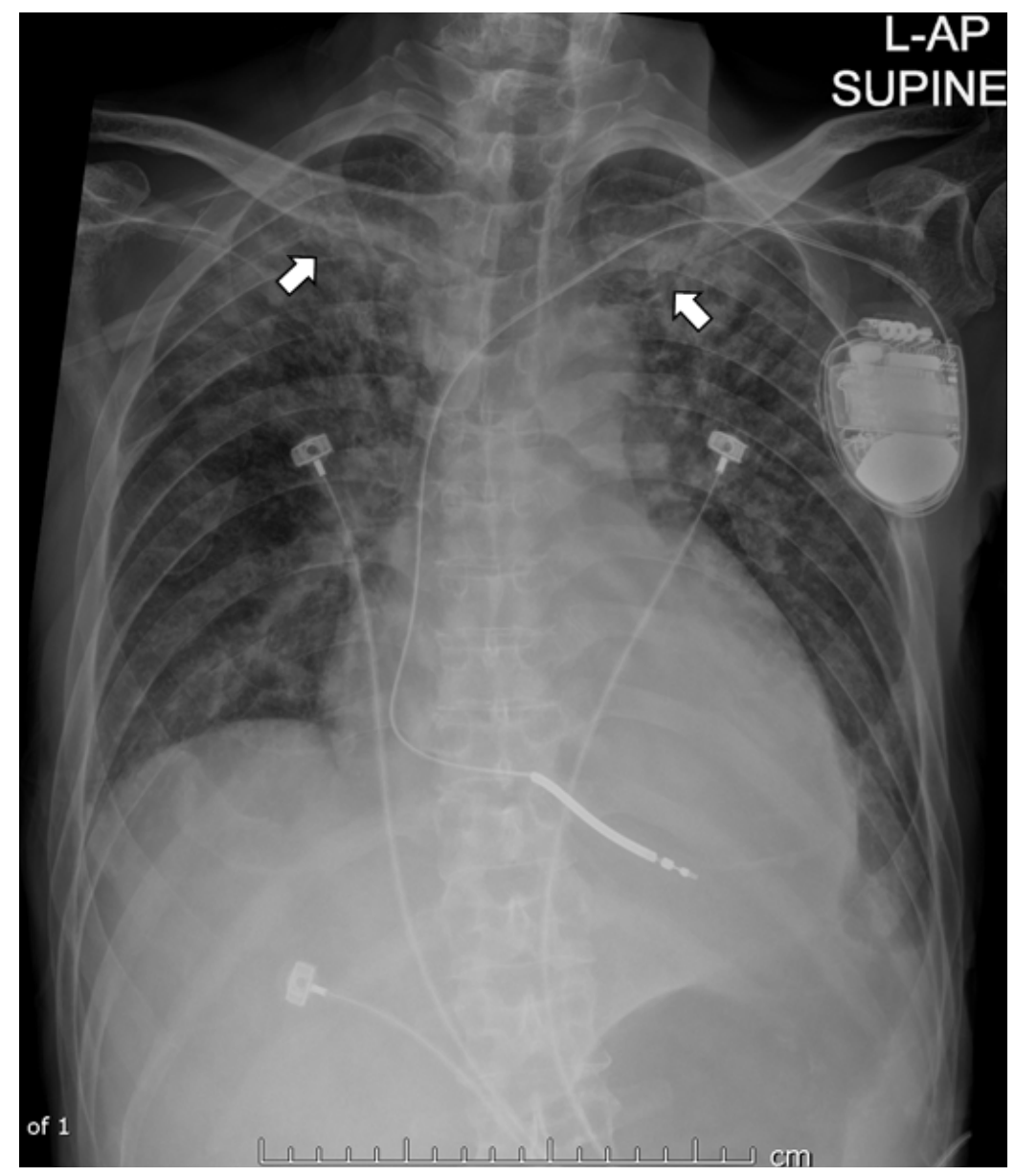

Figure 1. AP supine portable chest radiograph reveals diffuse patchy opacification with air bronchogram (white arrows) in both lungs, predominantly in both upper lobes. 


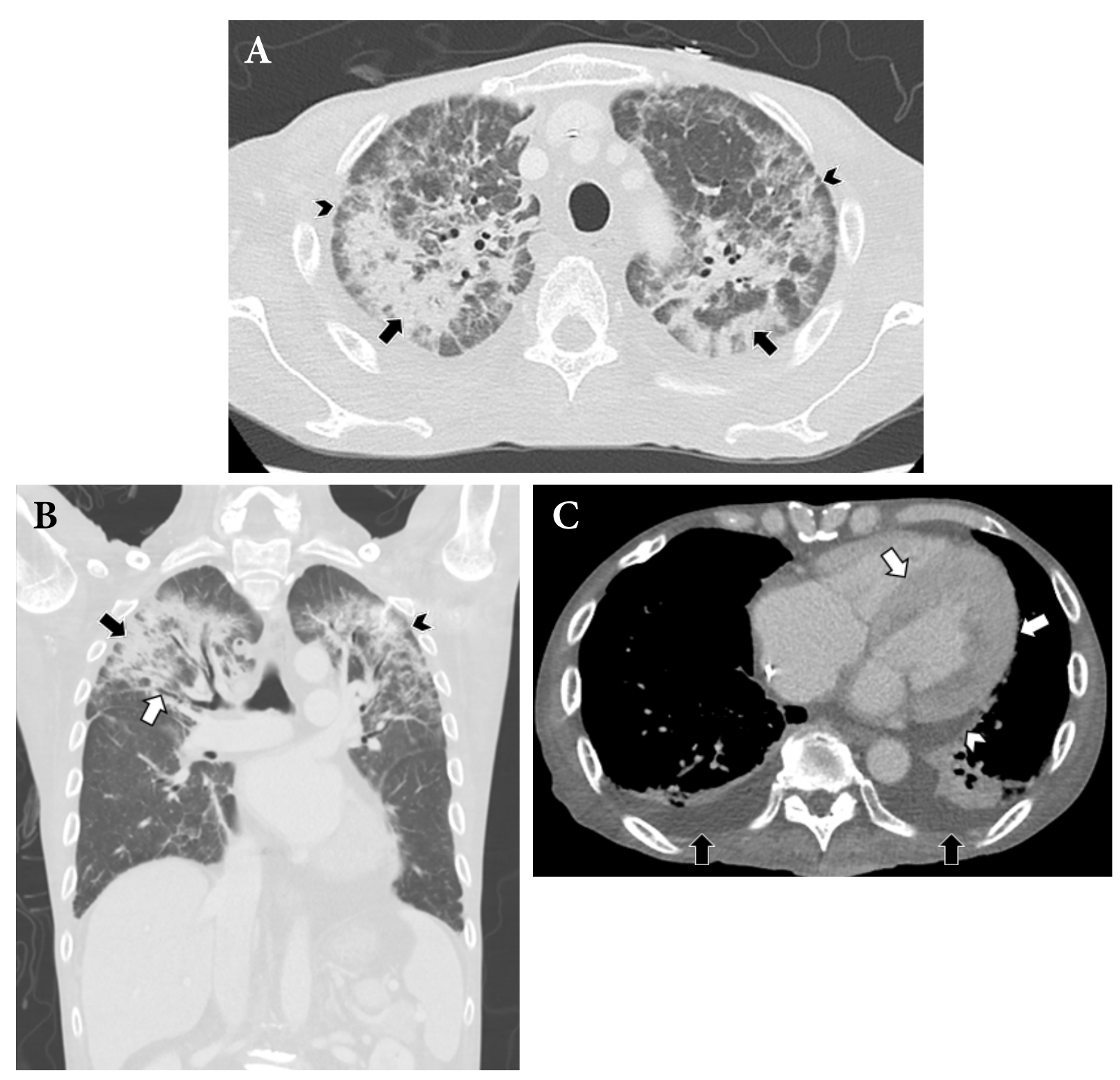

Figure 2. Venous phase chest computed tomography (CT).

$(A, B)$ Lung window axial and coronal images show bilateral pulmonary consolidations (black arrows), ground-glass opacities with air bronchograms (white arrows), and interlobular septal thickening (black arrowheads) predominantly in both upper lobes.

(C) Mediastinal window axial image shows bilateral pleural effusions (black arrows), a common finding of pleural involvement of amyloidosis. Minimal pericardial effusion (white arrowhead), as well as concentric and symmetric left ventricular wall and interventricular septal thickening (white arrows) was also observed in this case, a common yet nonspecific finding in cardiac amyloidosis. 

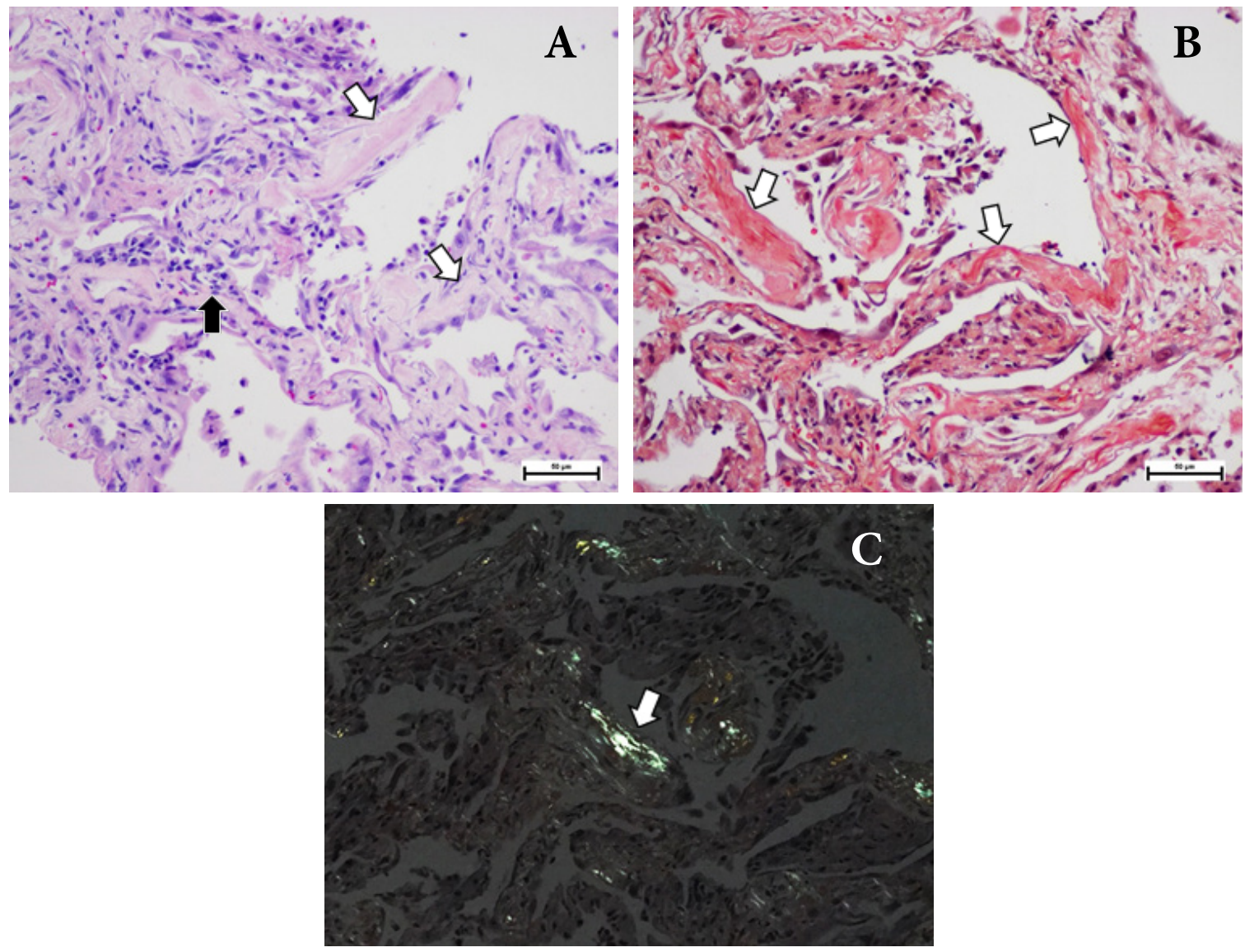

Figure 3. Microscopic examination of the right upper lobe lung tissue biopsy.

(A) The histology of the biopsied lesion shows benign alveoli with interstitial thickening with deposition of pink amorphous material (white arrows). The other area shows focal fibroblast proliferation with mild interstitial chronic inflammation (black arrows).

$(B, C)$ Congo red staining shows the area of abnormal eosinophilic material in the interstitial area $(B)$ and shows apple-green birefringence of the amyloid material in polarised light (C) (white arrows). 


\section{Discussion}

Pulmonary amyloidosis is a rare and lethal disease as it may cause pulmonary hypertension, respiratory failure, and eventually death. As described earlier, imaging findings of pulmonary amyloidosis are non-specific and the diagnosis of pulmonary amyloidosis still relies heavily on histopathological diagnosis. Previously reported imaging findings of nodular parenchymal amyloidosis includes solitary or multiple pulmonary nodules which may rarely calcify and cavitate, while diffuse alveolar septal amyloidosis may manifest as reticulonodular opacities, interlobar septal thickening, and confluent consolidations with basal and peripheral predominance in the lungs[1].

In the case of our patient, although his chest $\mathrm{CT}$ images revealed nonspecific findings as described, they do coincide with the previously reported findings of diffuse alveolar septal amyloidosis[1,6]. No nodules, calcifications, or cavitary lesions were detected in our case. The patient also had bilateral pleural effusions, which is the most common finding in pleural involvement of amyloidosis[1]. Although pulmonary amyloidosis usually involves basal and peripheral lungs, upper lung involvement of nodular parenchymal amyloidosis has been reported[7]. In addition, minimal pericardial effusion as well as concentric and symmetrical left ventricular wall and interventricular septal thickening was also observed in this case, a common yet nonspecific finding in cardiac amyloidosis $[1,8]$.

In retrospect, taking into account the patient's underlying medical conditions of light chain multiple myeloma and systemic amyloidosis, one might consider that the patient may have pulmonary amyloidosis as well. However, due to the patient's clinical presentation mimicking that of pneumonia and pulmonary oedema, combined with nonspecific imaging findings which support both differential diagnoses, pulmonary amyloidosis was initially not suspected. The diagnosis of pulmonary amyloidosis in this patient was made through diagnostic bronchoscopy with histopathological evaluation from tissue biopsy alone. Our case further highlights the importance of maintaining a high clinical suspicion for 
such a rare disease, especially in patients with underlying medical conditions with a high risk of pulmonary amyloidosis, and the importance of histopathological evaluation in cases of unresolving pneumonia.

\section{Conclusion}

We described a case of a 56-year-old male with a known history of multiple myeloma free light chain lambda with International Staging System (ISS) stage II, systemic amyloidosis and cardiac amyloidosis, diagnosed with biopsy-proven pulmonary amyloidosis manifesting as nonspecific imaging findings of the chest. The role of imaging studies in the diagnosis of pulmonary amyloidosis is still very limited due to nonspecific findings, but may help guide pneumologists in planning their bronchoscopy and location of tissue biopsy. The diagnosis in our case was made based on tissue biopsy with histopathological diagnosis alone which highlights the importance of maintaining a high clinical suspicion for such a rare disease, especially in patients with underlying medical conditions with a high risk of pulmonary amyloidosis, and the importance of histopathological evaluation in cases of unresolving pneumonia. 


\section{References}

1. Czeyda-Pommersheim F, Hwang M, Chen SS, Strollo D, Fuhrman C, Bhalla S. Amyloidosis: modern cross-sectional imaging. Radiographics 2015;35:138192. doi: 10.1148/rg.2015140179.

2. Baumann B, Salina D, Aboulhosn K. Clinical pulmonary amyloidosis presenting as lung cavitation with bronchiectasis: a case report. BCMJ 2019;61:344-8.

3. Blancas-Mejía LM, Ramirez-Alvarado M. Systemic amyloidoses. Annu Rev Biochem 2013;82:745-74. doi: 10.1146/annurev-biochem-072611-130030.

4. Li G, Han D, Wei S, Wang H, Chen L. Multiorgan involvement by amyloid light chain amyloidosis. J Int Med Res 2019;47:1778-86. doi: 10.1177/ 0300060518814337.

5. Lal A, Akhtar J, Khan MS, Chen Y, Yaron Goldman. Primary endobronchial amyloidosis: a rare case of endobronchial tumor. Respir Med Case Rep 2018;23:163-6. doi: 10.1016/j.rmcr.2018.02.007.

6. Georgiades CS, Neyman EG, Barish MA, Fishman EK. Amyloidosis: review and CT manifestations. Radiographics 2004;24:405-16. doi: 10.1148/ rg.242035114.

7. Lee SH, Ko YC, Jeong JP, Park CW, Seo SH, Kim JT, et al. Single nodular pulmonary amyloidosis: case report. Tuberc Respir Dis (Seoul) 2015;78:385-9. doi: 10.4046/trd.2015.78.4.385.

8. Oda S, Kidoh M, Nagayama Y, Takashio S, Usuku H, Ueda M, et al. Trends in diagnostic imaging of cardiac amyloidosis: emerging knowledge and concepts. Radiographics 2020;40:961-81. doi: 10.1148/rg.2020190069. 